Diabetes

\title{
Mehr kardiovaskuläre Ereignisse unter Sulfonylharnstoffen
}

Hintergrund: Endpunktstudien zeigten bisher keinen eindeutig positiven Effekt einer Senkung der Blutglukosewerte auf kardiovaskuläre und Gesamtmortalität. In einer aktuell publizierten Studie wurde untersucht, ob der Effekt auf kardiovaskuläre Endpunkte möglicherweise bei Verwendung verschiedener oraler Antidiabetika unterschiedlich ist.

Methoden: Für die retrospektive Kohortenstudie haben die Forscher die Daten der britischen UK general practice research database der Jahre 1990-2005 herangezogen. Ausgewertet wurden die Daten von 91521 Typ-2-Diabetikern in Bezug auf Inzidenz von Myokardinfarkt, Herzinsuffizienz und Gesamtmortalität. Berücksichtigt wurde dabei, welche Patienten über welchen Zeitraum mit welchen oralen Antidiabetika bzw. Insulin behandelt worden waren.

Ergebnisse: Im 15-jährigen Beobachtungszeitraum wurden 3588 Myokardinfarkte, 6900 Fälle von Herzinsuffizienz und 18548 Todesfälle berichtet. Im Vergleich

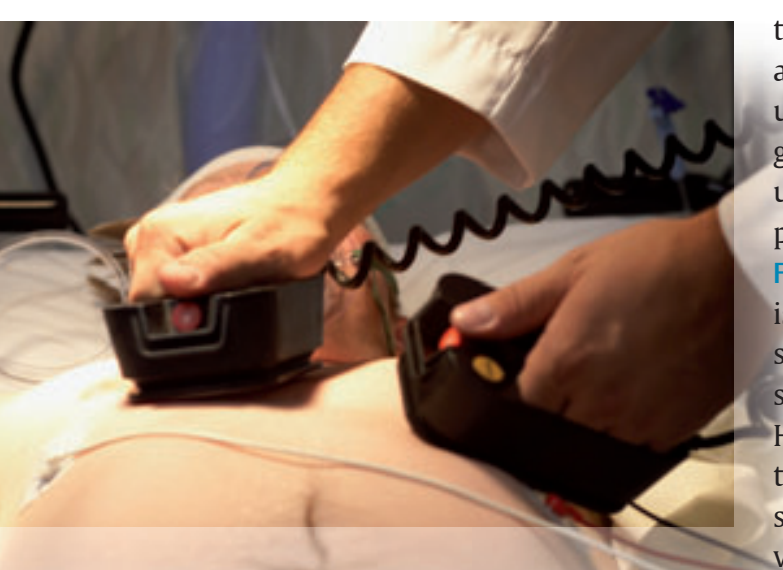

talität signifikant um 31-39\% abgesenkt $(\mathrm{p}=0,02$ bis $\mathrm{p}<0,001)$ und unter Rosiglitazon - im Vergleich zu Pioglitazon - signifikant um $34-41 \%$ erhöht $(p=0,14$ bis $\mathrm{p}=0,01)$.

Fazit: Im Vergleich zu Metformin ist der Einsatz von Sulfonylharnstoffen mit einer signifikanten Risikoerhöhung für Myokardinfarkt, Herzinsuffizienz und Gesamtmortalität assoziiert. In Bezug auf diese Endpunkte scheint nach der vorliegenden Analyse Pioglitazon zu Metformin war die Monotherapie mit. im Vergleich zu Rosiglitazon von Vorteil Sulfonylharnstoffen der 1. und 2. Genera- : zu sein. Die Autoren weisen ausdrücklich tion mit einer signifikanten 24-61\%igen a d auf die statistischen Limitationen der

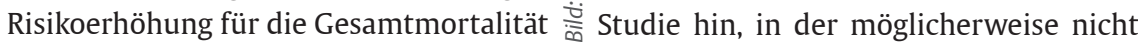
$(\mathrm{p}<0,001)$ und die Monotherapie mit Sulfonylharnstoffen der 2. Generation mit einer signifikanten 18-30\%igen Risikoerhöhung für eine Herzinsuffizienz ( $p=0,01$ und $p<0,001)$ assoziiert. Keinen signifikanten Einfluss auf die untersuchten Endpunkte hatte die Therapie mit Thiazolidindionen im Allgemeinen. Allerdings war unter Pioglitazon - im Vergleich zu Metformin - die Gesamtmoralle zugrundeliegenden Störfaktoren berücksichtigt werden konnten.

Dr. med. Winfried Keuthage, Münster

Quelle: Tzoulaki I, Molokhia M, Curcin V et al. Risk of cardiovascular disease and all cause mortality among patients with type 2 diabetes prescribed oral antidiabetes drugs: retrospective cohort study using UK general practice research database. BM] 2009; 339: b4731 